\title{
Hinode Flare Catalogue
}

\author{
K. Watanabe $\cdot$ S. Masuda $\cdot$ T. Segawa
}

Received: 10 December 2011 / Accepted: 25 March 2012 / Published online: 19 April 2012

(C) The Author(s) 2012. This article is published with open access at Springerlink.com

\begin{abstract}
We describe a catalogue of solar flares observed by the three instruments (SOT, XRT, EIS) onboard the Hinode satellite. From the launch of the Hinode satellite in September 2006 until late 2011, about 5000 solar flares (larger than A-class in the GOES classification) occurred during the five-year period of Hinode observations, and more than half of them were captured by the Hinode telescopes. Observation information for RHESSI and Nobeyama Radioheliograph are also included in the catalogue. This catalogue is distributed to users through the Internet. It will be useful and helpful for scientists in surveying flares to be analyzed, facilitate access to Hinode data, and help advance data analysis activities among the world solar community.
\end{abstract}

Keywords Catalogue $\cdot$ Sun: flare

\section{Introduction}

In recent years, various catalogue and event lists of observed solar flare data have been open to the public. These catalogue and lists have been extensively utilized by many researchers for finding data on individual flares as well as statistical research. For example, the previous Japanese solar mission, Yohkoh (Ogawara et al., 1991), had been operated for almost one complete solar cycle, and observed many solar phenomena, including flares. The catalogue of solar flares observed by Yohkoh (Sato et al., 2003, 2006) is still useful for scientists for surveying flares and has been used in many studies (e.g., Jakimiec and Tomczak, 2010).

\footnotetext{
K. Watanabe $(\bowtie)$ Institute of Space and Astronautical Science, Japan Aerospace Exploration Agency, 3-1-1 Yoshinodai, Chuo-ku, Sagamihara, Kanagawa 252-5210, Japan e-mail: watanabe.kyoko@isas.jaxa.jp

S. Masuda $\cdot$ T. Segawa

Solar-Terrestrial Environment Laboratory, Nagoya University, Furo-cho, Chikusa-ku, Nagoya 464-8601, Japan

S. Masuda

e-mail: masuda@stelab.nagoya-u.ac.jp
} 
Figure 1 The field of view (FOV) of Hinode. XRT has the capability to observe the full solar disk of $2048 \times 2048$ arcsec. EIS has a maximum FOV of $590 \times 1024$ arcsec, and SOT has a much smaller maximum FOV of $328 \times 164$ arcsec.

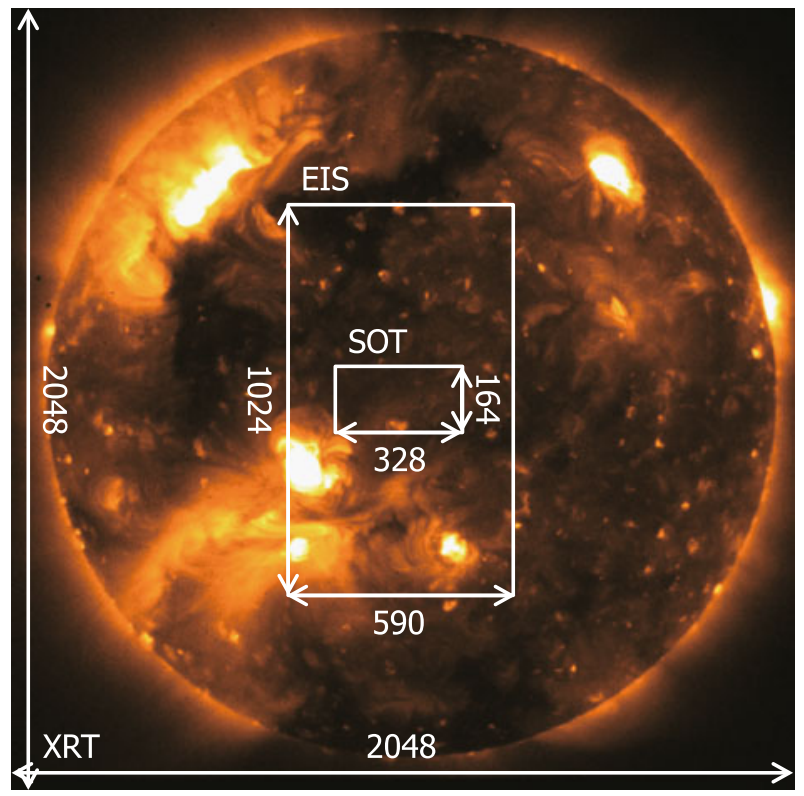

The Hinode satellite (Kosugi et al., 2007) has been in operation for almost five years, and already many flares have occurred during the Hinode era to date, even though still prior to solar maximum. Many of these flares were observed by Hinode. A catalogue of Hinodeobserved flares is now needed for statistical and other flare studies.

Except for soft X-ray images taken under the synoptic program, the field of view (FOV) of the Hinode instruments does not cover the full Sun. Namely, even if Hinode observes the Sun during a solar flare, that flare need not necessarily be observed by Hinode. Therefore, this Hinode Flare Catalogue will be useful for many researchers studying solar flares.

In this paper, we will introduce the Hinode Flare Catalogue, and the procedure for using it. We will also discuss the observability of solar flares by Hinode in Section 4.

\section{Field of View of the Hinode Instruments}

Hinode has three telescopes: the Solar Optical Telescope (SOT; Tsuneta et al., 2008; Suematsu et al., 2008; Ichimoto et al., 2008; Shimizu et al., 2008), the X-ray Telescope (XRT; Golub et al., 2007; Kano et al., 2008), and the EUV Imaging Spectrometer (EIS; Culhane et al., 2007). These instruments have different sizes of their field of view (FOV) as shown in Figure 1. XRT has the capability to observe the full solar disk of $2048 \times 2048$ arcsec. EIS has a maximum FOV of $590 \times 1024 \mathrm{arcsec}$, and SOT has a much smaller maximum FOV of $328 \times 164$ arcsec. Most of the time, the spacecraft pointing is not at the Sun center, and/or it uses a smaller FOV in order to maintain a good time cadence. The three telescopes usually coordinate their observations for studying the same target at which the spacecraft is pointed. The spacecraft pointing can be set at any location between the Sun center and the limb for observations of a specified target. Optionally, EIS has the ability to offset its own pointing with respect to the other two telescopes. Also, XRT, having a larger field of view than the others, has the ability to observe its own region of interest independent of the other two telescopes. 
Because of these FOV constraints, Hinode cannot observe all solar flares. Nonetheless, Hinode has been able to observe many flares, and it would be convenient to have ready-touse information on which flares Hinode did see. For this reason, we developed the Hinode Flare Catalogue. For a flare to be seen by one of the Hinode instrument, the flare must occur when that instrument is observing, and the flare must be inside that instrument's FOV. The catalog lists all flares during the five-year operation of Hinode. For each flare, we indicate whether the flare was seen by which of the Hinode instruments, or was not observed at all by Hinode.

\section{Procedure of the Hinode Flare Catalogue}

The procedure to make the Hinode Flare Catalogue is as follows:

i) Record all flares noted in the "Solar Software (SSW) latest image page" (http://www. Imsal.com/solarsoft/last_events/) of the Lockheed Martin Solar and Astrophysics Laboratory (LMSAL) during Hinode's operation period to date; this site contains position information for all flares that it lists.

ii) For each flare, check whether Hinode was observing during the time of the event, and if yes, whether the flare was in the Hinode FOV of any of the Hinode instruments. For these Hinode-observed events, count the number of images in

XRT: nine X-ray filter images,

SOT: filtergraph (FG) images,

EIS: all observed data.

iii) Compare with the flare list of the Reuven Ramaty High Energy Solar Spectroscopic Imager (RHESSI; Lin et al., 2000). For each solar flare that was also observed by RHESSI, check the maximum energy range of hard X-rays observed by RHESSI.

iv) Check whether the flare was also observed by the Nobeyama Radioheliograph (NoRH; Nakajima et al., 1994).

Almost all solar flares classified by the Geosynchronous Operational Environmental Satellite (GOES) are listed on the SSW Latest Image website, including class A flares (smallest GOES flares). This page gives the locations for almost all of the flares, based on EUV data from STEREO/SECCHI/EUVI, Solar and Heliospheric Observatory (SOHO)/EIT or Solar Dynamics Observatory (SDO)/AIA, and therefore it is very useful for determining whether Hinode observed the flare. We numbered all events sequentially from the first one during Hinode's observation day, which was on 20 October 2006. Event numbers in our initial list is in multiples of 10 , in order to retain the capability to add other events that are not listed as GOES flare events on the flare list of the SSW Latest Image page.

Next, for each flare, we determined whether it was inside the FOV of the Hinode telescopes. All data observed by the Hinode satellite are available from the website for the Data ARchive and Transmission System (DARTS; Miura et al., 2000; Matsuzaki et al., 2007). We use reformatted science data of Level 0 in DARTS for inspecting the Hinode data. We first obtained the data from the time of the flare (from GOES flare start time to end time). For EIS, we included all images that overlapped any part of the flare-occurrence period. We checked the location of the images on the Sun, and also the image sizes by using the header information of the FITS files. We then checked whether the images include a region of \pm 25 arcsec within the flare position on the SSW Latest Image page. Pointing offsets of these telescopes were occasionally made as mentioned by Shimizu et al. (2007), and some 
flares occurred near the edge of the FOV of the telescopes. Therefore, some events are not listed as Hinode-observed events despite being partially observed in some images. We used the following convention: If the flare position given in the SSW Latest Image page is outside of, but within 25 arcsec of, the FOV of one of the Hinode instruments, then we considered that event as observed by that instrument (but only as long as the image actually contains part of the flare, which we determine by visual inspection of the images). If Hinode observed the flare, we counted the number of images in which we saw the flare in XRT, SOT, and EIS data. For XRT, we choose X-ray images which were observed with at least one of its soft $\mathrm{X}$-ray filters (we did not consider images seen only with its G-band or one of its calibration filters). For SOT, we counted the number of images of FG data in the FG folder in DARTS as SOT flare images. We used all filtergram images in the FG folder, independent of whether they were from the Broad-band Filter Imager (BFI) or from the Narrow-band Filter Imager (NFI). No Spectro-Polarimeter (SP) images are included in the FG folder, and so SP images are not included in our list. For EIS, we checked all observed data, and counted the number of observed images.

Then, for each of the solar flares, we looked at the flare list of RHESSI (http://hesperia. gsfc.nasa.gov/hessidata/dbase/hessi_flare_list.txt), and checked the maximum energy range of hard X-rays observed by RHESSI.

Finally, we also checked for the existence of an observed event by NoRH, and added NoRH event number for commonly observed events.

The website for the Hinode Flare Catalogue is http://st4a.stelab.nagoya-u.ac.jp/hinode_ flare/. From the top page, a user can choose the list of all flares, or they can choose a monthly event list. The list provides the event number, GOES event time, location of the flaring region, the GOES X-ray class, and the number of observed images in SOT, XRT, and EIS, the maximum X-ray energy range observed by RHESSI, and NoRH event number. Clicking the event number brings up a summary plot of the observed images.

Figure 2 shows an example of a summary plot. In the summary plot, the top-left panel shows the GOES X-ray profile, and the top-right panel shows a sample X-ray image observed by XRT. The bottom-left panel shows a sample SOT flare image observed in Ca II $\mathrm{H}$, and the bottom-right panel shows an EIS Fe XII $195 \AA$ line EUV image. These sample images are taken from around the flare maximum time. In some summary plots, some panels say "No Data" in place of an image, if the flare was not seen at the specified wavelength. But even in cases where the panel says "No Data," there is a chance that some other wavelengths of SOT or EIS may have observed the flare. In such cases, the "observed image number" for the corresponding instrument in the event list will not be zero. For example, there may be no SOT Ca images for the event, but there may be SOT images at other wavelengths available.

\section{Statistics}

There have been 4784 solar flares between 20 October 2006 and 31 October 2011. Among them, 11 events were X-class; 123 events, or $2.6 \%$, were M-class; $27 \%$ were C-class; and more than $70 \%$ were B-class or smaller. This period covered solar minimum. Nonetheless, $3 \%$ of the events were M-class or larger. Table 1 gives the number of solar flares observed by each instrument in each year. The total number of flares varies following the solar cycle. Among all the flares, more than $50 \%$ were observed by XRT. Because of their smaller FOV, SOT and EIS saw fewer flares, but SOT still saw about $25 \%$ of all flares, and EIS saw $15 \%$.

Although the number of flares has been increasing recently, along with solar activity, the percentage observed by the instruments is almost the same. This is because many active 
$0036802006 / 12 / 13$ 02:14 - 02:40 - 02:57UT S07W22 X3.4
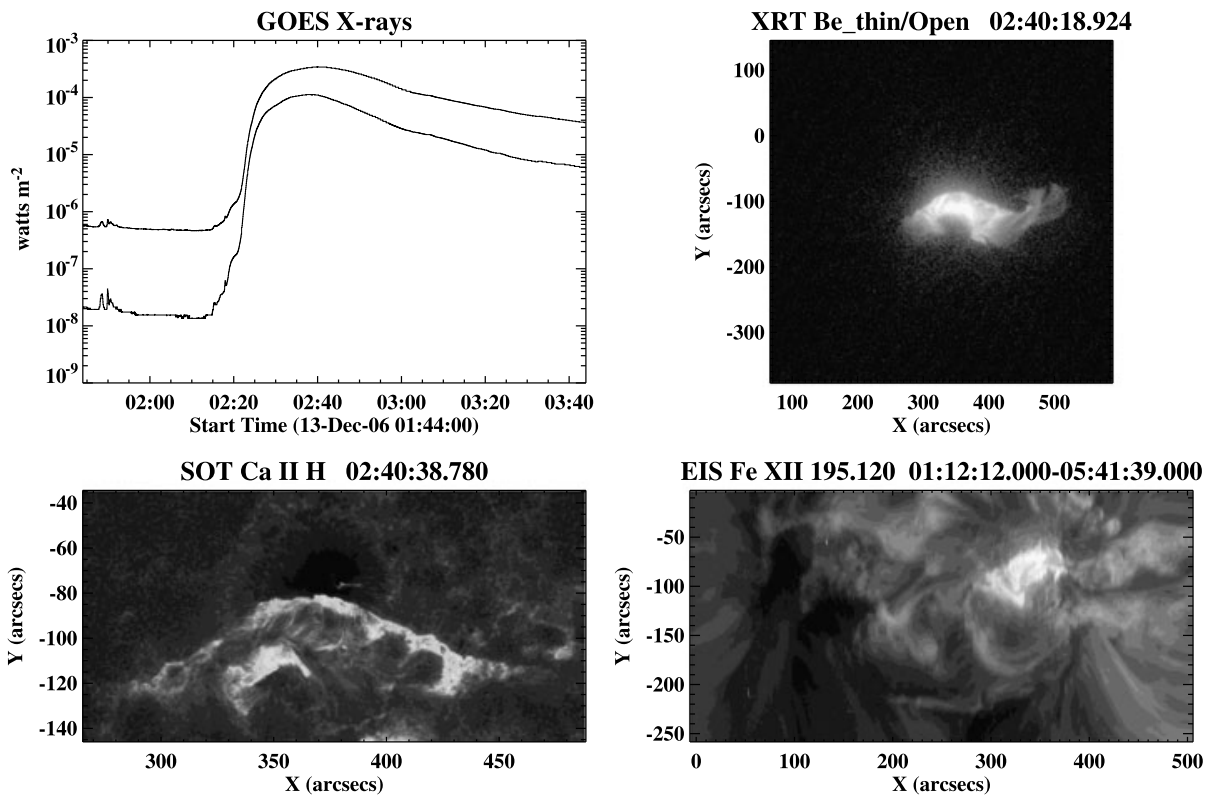

Figure 2 Example of a summary plot. The top-left panel shows the GOES X-ray profile, and the top-right panel shows an X-ray images observed by XRT. The bottom-left panel shows an SOT flare image observed at the Ca II H wavelength, and the bottom-right panel shows an EIS Fe XII $195 \AA$ A line EUV image.

Table 1 Number of solar flares observed by Hinode.

\begin{tabular}{lcccc}
\hline Year & XRT & SOT & EIS & Total flare no. \\
\hline 2006 (from 20 October) & 160 & 171 & 68 & 432 \\
2007 & 443 & 267 & 209 & 713 \\
2008 & 24 & 8 & 7 & 134 \\
2009 & 125 & 83 & 65 & 320 \\
2010 & 690 & 264 & 125 & 1248 \\
2011 (until 31 October) & 1019 & 377 & 237 & 1937 \\
Total event no. & 2461 & 1170 & 711 & 4784 \\
Observability & $51.4 \%$ & $24.5 \%$ & $14.9 \%$ & \\
\hline
\end{tabular}

regions are located on the solar surface, and so there is an increased chance that Hinode will point at a region other than that which flares. (During quieter times, there would often be a single active region on the Sun, and Hinode was likely to be observing it when it flared.)

In Table 1, an "observed" event assumes that there was at least one observed image. The user's scientific project may, however, require more than one image per event, depending on their specific study. To address this, we included a link to a csv file of the catalogue page; using this file, e.g., a user may easily filter out events with a specified minimum number of images (events with at least five images, for example). Similarly, they could filter this csv file for flare size, flares occurring near the limb, etc. 


\section{Summary}

The Hinode Flare Catalogue is available at http://st4a.stelab.nagoya-u.ac.jp/hinode_flare/. In addition to Hinode information, the catalog contains information on simultaneous RHESSI and NoRH observations. The catalogue will be updated monthly, with the most recent data being approximately one month old. This catalogue will be useful and helpful for scientists in surveying flares for analysis, facilitate access to Hinode data, and further data analysis activities among the world solar community.

Acknowledgements Hinode is a Japanese mission developed and launched by ISAS/JAXA, with NAOJ as domestic partner and NASA and STFC (UK) as international partners. It is operated by these agencies in co-operation with ESA and NSC (Norway). A part of this work was carried out with the support of a joint research program grant of the Solar-Terrestrial Environment Laboratory, Nagoya University. We thank T. Shimizu and S. Imada for providing information that was essential to develop the Hinode Flare Catalogue. We also thank A. Sterling for helpful discussions.

Open Access This article is distributed under the terms of the Creative Commons Attribution License which permits any use, distribution, and reproduction in any medium, provided the original author(s) and the source are credited.

\section{References}

Culhane, J.L., Harra, L.K., James, A.M., Al-Janabi, K., Bradley, L.J., Chaudry, R.A., et al.: 2007, Solar Phys. 243, 19.

Golub, L., DeLuca, E., Austin, G., Bookbinder, J., Caldwell, D., Cheimets, P., et al.: 2007, Solar Phys. 243, 63.

Ichimoto, K., Lites, B., Elmore, D., Suematsu, Y., Tsuneta, S., Katsukawa, Y., et al.: 2008, Solar Phys. 249, 233.

Jakimiec, J., Tomczak, M.: 2010, Solar Phys. 261, 233.

Kano, R., Sakao, T., Hara, H., Tsuneta, S., Matsuzaki, K., Kumagai, K., et al.: 2008, Solar Phys. 249, 263.

Kosugi, T., Matsuzaki, K., Sakao, T., Shimizu, T., Sone, Y., Tachikawa, S., et al.: 2007, Solar Phys. $243,3$.

Lin, R.P., Dennis, B.R., Hurford, G.J., Smith, D.M., Zehnder, A., Harvey, P.R., et al.: 2000, Solar Phys. $\mathbf{2 1 0}, 3$.

Matsuzaki, K., Shimojo, M., Tarbell, T.D., Harra, L.K., Deluca, E.E.: 2007, Solar Phys. $243,87$.

Miura, A., Shinohara, I., Matsuzaki, K., Nagase, F., Negoro, H., Uno, S., et al.: 2000, In: Manset, N., Veillet, C., Crabtree, D. (eds) Astronomical Data Analysis Software and Systems (ADASS) IX, ASP Conf. Ser. 216, 180.

Nakajima, H., Nishio, M., Enome, S., Shibasaki, K., Takano, T., Hanaoka, et al.: 1994, Proc. IEEE 82, 705.

Ogawara, Y., Takano, T., Kato, T., Kosugi, T., Tsuneta, S., Watanabe, T., Kondo, I., Uchida, Y.: 1991, Solar Phys. 136, 1.

Sato, J., Sawa, M., Yoshimura, K., Masuda, S., Kosugi, T.: 2003, The YOHKOH HXT/SXT Flare Catalogue, Montana State University, Bozeman, and The Institute of Space and Astronautical Science, Sagamihara, Japan.

Sato, J., Matsumoto, Y., Yoshimura, K., Kubo, S., Kotoku, J., Masuda, S., et al.: 2006, Solar Phys. $236,351$.

Shimizu, T., Katsukawa, Y., Matsuzaki, K., Ichimoto, K., Kano, R., DeLuka, E.E., et al.: 2007, Publ. Astron. Soc. Japan 59, 845.

Shimizu, T., Nagata, S., Tsuneta, S., Tarbell, T., Edwards, C., Shine, R., et al.: 2008, Solar Phys. $249,221$.

Suematsu, Y., Tsuneta, S., Ichimoto, K., Shimizu, T., Otsubo, M., Katsukawa, Y., et al.: 2008, Solar Phys. 249, 197.

Tsuneta, S., Suematsu, Y., Ichimoto, K., Shimizu, T., Otsubo, M., Nagata, S., et al.: 2008, Solar Phys. 249, 167. 